\title{
Öffentlich-private Partnerschaften : Bedeutung und Herausforderungen für die Entwicklungszusammenarbeit
}

Catherine Schümperli Younossian and Edouard Dommen

\section{CpenEdition}

Electronic version

URL: http://journals.openedition.org/sjep/464

DOI: $10.4000 /$ sjep.464

ISSN: 1663-9677

\section{Publisher}

Institut de hautes études internationales et du développement

Printed version

Date of publication: 1 octobre 2005

Number of pages: 11-21

ISBN: 2-88247-059-2

ISSN: $1660-5926$

\section{Electronic reference}

Catherine Schümperli Younossian und Edouard Dommen, «Öffentlich-private Partnerschaften :

Bedeutung und Herausforderungen für die Entwicklungszusammenarbeit », Schweizerisches Jahrbuch für Entwicklungspolitik [Online], 24-2 | 2005, Online erschienen am: 08 Juni 2010, abgerufen am 24 September 2020. URL : http://journals.openedition.org/sjep/464 ; DOI : https://doi.org/10.4000/sjep. 464 


\title{
Öffentlich-private Partnerschaften: Bedeutung und Herausforderungen für die Entwicklungszusammenarbeit
}

\author{
Catherine Schümperli Younossian* \\ in Zusammenarbeit mit Edouard Dommen**
}

\begin{abstract}
ie Auseinandersetzung mit den öffentlich-privaten Partnerschaften (PPP) in der Entwicklungszusammenarbeit hat sich als sehr delikate und heikle Aufgabe erwiesen. Wegen der unterschiedlichen Bedeutungen lässt sich das Konzept schwer umreissen; ausserdem sind festgefahrene und emotionsgeladene Positionen zu beobachten. Es gibt keine anerkannte Definition des Konzeptes PPP, welche den verschiedenen Akteuren der Entwicklungszusammenarbeit als Referenz dienen könnte; überdies ist der Begriff ungenau, weil er sich auf mehrere Analyseebenen bezieht.
\end{abstract}

\section{PPP: Definition gesucht}

Die erste Ebene betrifft das neue Entwicklungsparadigma, wonach Partnerschaften mit dem privatwirtschaftlichen Sektor gefördert werden sollen, was einen innovativen Rahmen schafft. Drei wesentliche Elemente verhalfen dieser neuen Ausrichtung zum Durchbruch. Zu Beginn der 90er Jahre machte sich die Weltbank als erste für PPP, besonders im Infrastrukturbereich, stark. Diese Option wurde sehr rasch von den bilateralen Entwicklungsorganisationen übernommen, die sich an der Umsetzung des ,integrierten Rahmens für die Entwicklung“ beteiligten. Im Jahr 1999 lancierte der UNO-Generalsekretär Kofi Annan anlässlich des Weltwirtschaftsforums in Davos den „Global Compact“, welcher die Verwirklichung der sozialen und wirtschaftlichen Rechte und der Umweltnormen durch die Unternehmen vorantreiben soll. Schliesslich betonten die Regierungen anlässlich der Konferenz von Monterrey über die Entwicklungsfinanzierung im Jahr 2002 die Schlüsselrolle der PPP als Instrument zur Mobilisierung neuer Ressourcen, um sich den Millenniumszielen anzunähern. Angesichts der stagnierenden bzw. sogar rückläufigen öffentlichen Entwicklungshilfe führt kein Weg an neuen Partnerschaften vorbei.

Die zweite Ebene betrifft die vielfältigen Beziehungen zwischen internationalen Organisationen und Privatunternehmen. Die unterschiedlichsten Initiativen tragen das Label PPP: der Global Compact, Sponsoring-Arbeit oder gemeinsame Projekte. Die Fallbeispiele für eine multilaterale öffentlich-private Partnerschaft (MPPP) im umseitigen Rahmentext 1 sollen diese Ebene veranschaulichen. Abgesehen davon befasst sich das vorliegende Werk jedoch nicht mit dieser Art PPP.

* Forschungs- und Lehrbeauftragte am IUED, verantwortlich für das Dossier.

** Präsident des Wissenschaftlichen Ausschusses des RUIG (Internationales Universitätsnetz von Genf). Edouard Dommen ist der Verfasser der Rahmentexte. 


\section{Rahmentext 1}

\section{Zwei Beispiele für eine multilaterale öffentlich-private Partnerschaft (MPPP)}

Global Alliance for Vaccines and Immunization (GAVI)

(Weltweite Allianz für Impfstoffe und Immunisierung)

Im Rahmen der GAVI werden die Partner den folgenden Gruppen zugeordnet:

- Regierungen der Industrieländer;

- Regierungen der Entwicklungsländer;

$-\mathrm{NGO}$;

- internationale Organisationen (besonders WHO, UNICEF und Weltbankgruppe);

- eine Stiftung: die Stiftung Bill und Melinda Gates

- Institute für Forschung und öffentliche Gesundheit;

- Impfstoffindustrie der Industrieländer (die Unternehmen sind in der International Federation of Pharmaceutical Manufacturers Associations IFPMA (Weltverband der pharmazeutischen Industrie) zusammengeschlossen;

- Impfstoffindustrie der Entwicklungsländer (es handelt sich um eine Ad-hoc-Berufsvereinigung).

Website der GAVI : <www.vaccinealliance.org $>$.

Global Public-Private Partnership for Handwashing with Soap

(Globale öffentlich-private Partnerschaft für Händewaschen mit Seife)

Die bei der Weltbank in Washington angesiedelte PPP zählt folgende Mitglieder:

- internationale Organisationen: Weltbank, UNICEF;

- Dienststellen der Bundesregierung der Vereinigten Staaten;

- mehrere der weltweit grössten Seifenproduzenten: Colgate-Palmolive, Procter and Gamble, Unilever, Hindustan Lever;

- eine US-amerikanische NGO;

- eine akademische Institution: die London School of Hygiene and Tropical Medicine.

Website der Partnerschaft: <www.globalhandwashing.org $>$.

Die dritte Variante der PPP schliesslich besteht in der neuen Rollenverteilung zwischen öffentlichem und privatem Sektor bei der Erfüllung bestimmter Entwicklungsaufgaben.

Zur Klärung des Konzepts und zur methodischeren Definition der PPP formulierten wir drei Fragen, die den roten Faden des vorliegenden Werks bildeten und an denen sich Autoren in ihren Überlegungen orientierten:

口 Wie ist die neue Praxis in der internationalen Zusammenarbeit entstanden?

口 Welche Potenziale, Risiken und Grenzen besitzen die PPP?

$\checkmark$ Unter welchen Rahmenbedingungen lassen sich die positiven Wirkungen der PPP verstärken und gleichzeitig die unerwünschten Wirkungen verringern ?

Das Werk, das sich hauptsächlich mit der ersten und dritten Ebene befasst, verfolgt den Zweck, eine Diskussion einzuleiten, in welcher die interessierten Kreise die verwendeten Konzepte vertiefen und klären können, und soll auch die unterschiedlichen Erwartungen an die PPP verdeutlichen. Das Komitee des Schweizerischen Jahrbuchs für Entwicklungspolitik wünscht sich, dass das Dossier zur „Entideologisierung“ der Diskussion, die teilweise zu stark von Vorurteilen über die Stärken und Schwächen des Privatsektors und der öffentlichen Hand belastet war, beitragen kann. 


\section{Rahmentext 2}

\section{„Öffentlich-private Partnerschaft": ein Triggerwort}

PPP gilt als Triggerwort: Solche Wörter verweisen nicht auf analytische Konzepte. Wer das jedoch kritisiert, hat die Funktion der Triggerwörter falsch verstanden: Triggerwörter sollen Reaktion hervorrufen und bestimmte Haltungen orientieren; sie sollen zusammenführen und mobilisieren. Die Äusserungen von Serge Latouche über das Oxymoron lassen sich auch auf das Triggerwort beziehen: Die „Erfindung der Dichter, um das Unsägliche zu sagen, wird zunehmend von den Technokraten benutzt, um das Unglaubliche glaubhaft zu machen"a. Die emotionalen und kulturellen Gedankenverbindungen der Triggerwörter sind demnach dem semantischen Inhalt übergeordnet.

Die Hauptaufgabe der Triggerwörter ist, eine Anziehungskraft und eine einigende Kraft auszuüben. Für die Attraktivität werden emotional besetzte Wörter verwendet, welche Sympathie wecken - wie im vorliegenden Beispiel „Partnerschaft". Zur Einigungsfunktion müssen die ausgewählten Wörter medizinisch gesprochen ein „Breitbandspektrum” aufweisen. Das Ideal ist es, Widersprüche in sich zu benennen: Niemand fühlt sich als Fremder, wenn dieses Wort nichts ausschliesst.

Triggerwörter sind relativ zahlreich, so dass allgemein eine Beschreibung des Lebenszyklus dieser Kategorie erstellt werden kann. Zunächst gibt das Triggerwort einen momentanen Wunsch wieder, der um Assoziationen und um emotional positive Zwischentöne bereichert wird. Um weitgehende Akzeptanz für das Wort zu gewinnen, betonen die Anhänger die Resonanzen und bemühen sich, die affektiven Aspekte besser zu nutzen; sie befrachten das Triggerwort mit den verschiedensten wünschenswerten Ideen. Mit dem Wort "Partnerschaft" wird so implizit ausgesagt, dass die Teilnehmer sich gegenseitig Respekt beweisen, eine bestimmte Gleichstellung untereinander erfahren und dass sie sich gemeinsam für ein geteiltes Ziel engagieren.

Die Anhänger des Slogans bemühen sich auch, analytische Inhaltselemente, welche Ablehnung auslösen könnten, aus dem Triggerwort zu entfernen. Jede Beschreibung eines Sachverhalts spaltet die Zuhörer in zwei Lager: die Befürworter und die Gegner, welche die Beschreibung als unrichtig beurteilen.

Fachleute halten es für notwendig, dem Triggerwort eine eindeutige technische Bedeutung zuzuordnen, um es als Arbeitsinstrument verwenden zu können. Deswegen bemühen sie sich um die Definition eines analytischen Inhalts. Es ist zu betonen, dass der Vorgang der Operationalisierung des Konzepts parallel und simultan zum Vorgang der Sinnentleerung erfolgt.

Je stärker das Konzept in ein Korsett gezwängt wird, desto weniger spricht es die Phantasie an und desto mehr verliert es an emotionalem Reiz. Operationelle Definitionen stossen in jedem Fall bei denjenigen, die unterschiedliche Konzeptumsetzungen oder Analysestrukturen befürworten, auf Widerstand. Je präziser der Inhalt eines Wortes definiert wird, desto weniger Freunde zählt das Wort !

Kurz, ein Triggerwort kann auf zwei Weisen aussterben: Entweder gewinnt die emotionale Dynamik die Oberhand und das Wort büsst so stark an Sinn ein, dass es an Leblosigkeit zu Grunde geht, oder der technische Inhalt setzt sich durch und das immer präzisere Wort macht sich so viele Feinde, dass man es vernichtet. Dem Wort „öffentlich-private Partnerschaft" dürfte eine kurze Lebensdauer beschieden sein: Es hat sich erst ab Anfang der 90er Jahre verbreitet, nähert sich aber bereits der Schlussphase.

a Serge Latouche, „Le développement peut-il être ,durable'?”, L'Ecologiste, Nr. 6, Winter 2001, S. 38-39.

Edouard Dommen 
Das vorliegende Werk ist in vier Teile gegliedert. Der erste Teil unter dem Titel „Entstehungsgeschichte und Umrisse des Konzeptes“ schildert die Voraussetzungen und Ursachen für das Aufkommen des Konzeptes in der Entwicklungszusammenarbeit und bietet dem Leser Elemente, um das Konzept zu umreissen. Der Begriff „öffentlich-private Partnerschaft“ hat ab Anfang der 90er Jahre Einzug in den Wortschatz der Entwicklungszusammenarbeit gehalten. Stimmt es aber, dass die Zusammenarbeit zwischen privatem und öffentlichem Sektor eine neue Erscheinung darstellt? Diese Interaktionen existierten in unterschiedlicher und vielfältiger Form seit je. Neu daran ist, dass die Wechselwirkungen einem „,neuen politischen Paradigma“ zuzuordnen sind, das diese Partnerschaften fördern soll. Frédéric Lapeyre vermittelt einen Überblick über die wesentlichen Etappen in der Geschichte der Entwicklungszusammenarbeit und untersucht die seit kurzem umgesetzten Massnahmen wie z.B. der „,integrierte Rahmen für die Entwicklung“ und die „Armutverringerungsstrategien“, denen die internationalen Finanzinstitutionen das Wort reden. Gemäss dem Autor „,verkörpern“ diese neuen Massnahmen das Entwicklungsparadigma, welches die Rollen- und Aufgabenverteilung zwischen staatlichen und nichtstaatlichen Akteuren grundlegend umgestellt hat. Der Artikel soll zeigen, dass die Umsetzung der PPP die Bewältigung der Interessenkonflikte zwischen den verschiedenen Stakeholders erfordert; Dialog und Verhandlung sind erforderlich, um Meinungsunterschiede zu überwinden und zu einem Kompromiss zu gelangen. Damit tragfähige Entwicklungsprojekte verwirklicht werden können, ist es unbedingt notwendig, die Beteiligung der Zivilgesellschaft und besonders die Rolle der Bevölkerung und ihrer repräsentativen Organisationen anzuerkennen.

Mit dem Begriff „Partnerschaft“ wird die Existenz von mindestens zwei unterschiedlichen, aber ebenbürtigen - Partnern mit sich gegenseitig ergänzenden Aufgaben, die sinnvollerweise auf ein gemeinsames (zu definierendes) Ziel hin arbeiten, implizit anerkannt. Auf der Basis dieser theoretischen Feststellung beleuchten Peter Ulrich und Florian Wettstein die Motivationssysteme der Protagonisten der PPP im Bereich der Entwicklungspolitik. Die Autoren beschreiben die Perspektiven der Entwicklungsethik, welche die Unternehmen befolgen müssen: Sie sollten als festen Bestandteil ihrer legitimen Anstrengungen zur Gewinnmaximierung soziale und öffentliche Verantwortlichkeiten übernehmen. Zur Veranschaulichung der Aussage schliesst der Artikel mit einer kritischen Analyse des unter der Ägide der Vereinten Nationen umgesetzten Global Compact.

Der vorhergehende Artikel hinterfragt die grundlegenden Prinzipien, denen der öffentliche und der private Bereich gehorchen; die unterschiedliche Zusammensetzung einer PPP kann je nach den betroffenen Akteuren die Art und den Geist der Partnerschaft deutlich beeinflussen. Die im Rahmentext 3 vorgestellte „Taxonomie“ von Edouard Dommen erweist sich als hilfreiches Instrument, um die Motivationen der potenziellen Partner feinrastrig zu analysieren. Um den Leser und die Leserin durch die Beiträge des Dossiers zu leiten, haben wir darin die Artikel, in denen die Rolle bzw. die Tätigkeiten der erwähnten Partner vertieft werden, jeweils mit dem Namen der Autoren identifiziert. 


\section{Rahmentext 3}

\section{Bestandteile einer öffentlich-privaten Partnerschaft}

Um als öffentlich-privat zu gelten, muss eine Partnerschaft aus mindestens zwei Partnern bestehen, von denen einer zur Kategorie der offiziellen Organisationen (öffentlicher Sektor) und der andere zum Sektor der Privatunternehmen oder der Zivilgesellschaft zählt.

\section{1. Öffentlicher Sektor}

- International: Dazu gehören insbesondere die UNO-Organisationen (WHO, UNICEF, Organisation der Vereinten Nationen für Bildung, Wissenschaft und Kultur [UNESCO]...) oder die Bretton-Woods-Institutionen und die Entwicklungsbanken.

[D. Dossier PPP: F. Lapeyre, P. Ulrich/ F. Wettstein, J. Richter.

- National: Dazu gehören der Staat mit der zivilen und militärischen Komponente sowie gegebenenfalls die Staatsunternehmen.

[D. Dossier PPP: B. Chassot Gétaz/K. von Däniken/T. Zeller (seco/DEZA), W. Gruber/D. Rothenberger (seco), S. Cohen (Staat Genf), X. Tschumi Canosa, G. Carbonnier.

- Lokal: Dazu gehören die öffentlichen Behörden der verschiedenen Staatsebenen (Provinzen, Kommunen, usw.) und gegebenenfalls die angeschlossenen Unternehmen.

[e] Dossier PPP: M. Tidjani Alou.

\section{Sektor der Privatunternehmen}

- Unternehmen:

- Transnationale Unternehmen können eine Partnerschaft auf der Ebene der Zentralstelle, einer nationalen Tochtergesellschaft oder einer Einrichtung (z.B. Fabrik oder Zweigniederlassung) an einem bestimmten Ort bilden ;

- grosse nationale Gesellschaften;

- KMU: ausländische oder inländische Unternehmen.

[Dol Dossier PPP: S. Koch.

- Berufsverbände: Internationale, nationale oder lokale Berufsverbände umfassen Organisationen, die mehrere Branchen (z.B. Handelskammern) oder einen bestimmten Sektor (Fremdenverkehrsamt) abdecken, oder Vereinigungen, zu denen mehrere Unternehmen der gleichen Branche gehören (z.B. Weltverband der pharmazeutischen Industrie, IFPMA).

[Da Dossier PPP: S. Cohen.

- Wohltätige Unternehmenseinrichtungen: Mehrere Unternehmen führen Firmenstiftungen (z.B. "Novartis Foundation for Sustainable Development" oder sonstige wohltätige Strukturen (z.B. Nestlé, siehe <www.community.nestle.com>)

\section{Zivilgesellschaft}

- Die NGO sind gleichberechtigte Partner zahlreicher PPP.

- Die Gemeinschaften umfassen auch Stellen der Zivilgesellschaft, die keine öffentlichen Behörden sind (z.B. Quartiervereine).

[Da Dossier PPP: S. Hoffmann, C. Eberlein.

- Die wohltätigen Einrichtungen (abgesehen von den Unternehmensstiftungen) decken eine breite Palette von Institutionen ab. Die Stiftung Bill und Melinda Gates spielt besonders im Rahmen von PPP im Gesundheitsbereich, z.B. im Weltfonds zur Bekämpfung von AIDS, Tuberkulose und Malaria, eine aktive Rolle. Die Unternehmensstiftung Fundación Aguas Argentinas, der verlängerte Arm einer PPP, arbeitet mit staatlichen Institutionen und mit gemeinschaftlichen Organisationen zusammen.

4. Akademische Institutionen und Forschungseinrichtungen

5. Weitere mögliche Partner 
Im zweiten Teil des Werks, „Die öffentlich-privaten Partnerschaften und die Entwicklungszusammenarbeit“, werden die PPP als Mittel der Entwicklungszusammenarbeit vorgestellt. Die PPP werden hinsichtlich ihrer operationellen Dimension als Bündnis zwischen staatlichen und privaten Einheiten wahrgenommen, die zur Erreichung eines gemeinschaftlichen Ziels zusammenspannen. Auf diese Weise soll die Durchführung eines Projekts von öffentlichem Interesse unter der gegenseitigen Verantwortung eines öffentlichen und eines privaten Akteurs verbessert werden. Die Finanzierung wird ebenfalls durch gemeinsame (private und öffentliche) Mittel gesichert und in einem Vertrag geregelt. In bestimmten Fällen sind die PPP zu einem Instrument für die von internationalen Institutionen geförderte Privatisierungspolitik geworden. In anderen Situationen ermöglichen sie die Zusammenarbeit zwischen komplementären Partnern für die Umsetzung von Entwicklungsprojekten. Wie bereits erwähnt, ist dieser Ansatz in einem Umfeld der Stagnation oder gar des Rückgangs der öffentlichen Entwicklungshilfe entstanden. So konnten neue Finanzierungsquellen erschlossen werden.

Die Schweizer Entwicklungszusammenarbeit beteiligt sich über die Direktion für Entwicklung und Zusammenarbeit (DEZA) sowie das Staatssekretariat für Wirtschaft (seco) an PPP-Projekten und leistet entsprechende Unterstützung. In einem ersten gemeinsamen Artikel von DEZA und seco plädieren Brigitte Chassot Gétaz, Kathi von Däniken und Thomas Zeller für eine verstärkte Zusammenarbeit zwischen öffentlicher Hand und Privatwirtschaft. Dies hätte den wesentlichen Vorteil eines Multiplikatoreffekts und eines langfristigen Wirtschaftswachstums. Die beiden wichtigsten Schweizer Einrichtungen für Entwicklungszusammenarbeit verfolgen zwar auf diesem Weg dasselbe Ziel (Linderung der Armut und Beitrag zur Erreichung der Millenniums-Entwicklungsziele), verfügen aber über ihre eigenen Modalitäten und Leitlinien für die Zusammenarbeit mit der Privatwirtschaft. Im Bereich der PPP hat sich das seco auf Infrastrukturen spezialisiert. Die detailreiche Präsentation von Werner Gruber und Dieter Rothenberger bietet einen Einblick in die Ziele und Mittel des seco anhand von Beispielen wie der Stromversorgung in Tadschikistan oder der PPP im Bereich Wasserversorgung in Tansania und Bolivien.

Für eine erweiterte Sichtweise geht Xavier Tschumi Canosa auf die Praxis der deutschen und der französischen Entwicklungszusammenarbeit ein und unterstreicht dabei die zunehmende Bedeutung der gemäss diesen Modalitäten durchgeführten Projekte. Der Autor weist auf die Vorteile der PPP bei der Beschaffung zusätzlicher Mittel zur Erreichung der Millenniums-Entwicklungsziele hin, betont jedoch die Bedeutung eines soliden institutionellen Rahmens in den Entwicklungsländern, um möglichen Missbräuchen durch die beteiligten Partner vorzubeugen. Ausserdem hebt er die Vorrangigkeit des Entwicklungsziels hervor, für das die Entwicklungsagenturen bürgen müssen.

Schliesslich erhält auch Sylvie Cohen (Leiterin der Direktion für auswärtige Angelegenheiten des Kantons Genf) das Wort. Sie präsentiert die Entwicklungszusammenarbeit ihres Kantons. Dieser hat sich in jüngster Zeit mit den notwendigen Mitteln ausgestattet, um von Genfer Privatunternehmen vorgeschlagene Entwicklungsprojekte zu unterstützen. 
Im dritten Teil, „Probleme und Herausforderungen. Standpunkte verschiedener Akteure", erhalten Vertreter von NGO, aus dem Privatsektor und Akademiker das Wort. Besondere Beachtung finden dabei die PPP im Zusammenhang mit der Wasserversorgung: Über die Hälfte der Artikel dieses Dossiers befassen sich mit PPP in diesem Bereich und bieten differenzierte Einblicke sowie zahlreiche Beispiele ${ }^{1}$. Sie greifen die Äusserungen von Frédéric Lapeyre auf, der im ersten Artikel des Werks darauf hinweist, dass es heute in den Sektoren Wasserversorgung und Abwasserentsorgung mehr als 2300 PPP gibt. Zu Beginn der 90er Jahre waren praktisch noch keine solchen Projekte zu verzeichnen. Der Autor stellt fest, dass die Förderung der Partnerschaften und die Neufestlegung der Verantwortlichkeiten zwischen öffentlichen und privaten Akteuren nicht immer unproblematisch sind, weil diese Politik zur Verbesserung der Qualität und des Zugangs zu den Grundversorgungsdiensten im Zusammenhang mit ihrer Privatisierung manchmal umstrittene Ergebnisse gezeitigt hat.

Christine Eberlein beleuchtet die PPP kritisch und stellt die Frage nach den Möglichkeiten des Zugangs zu den Infrastrukturen für die unter der Armutsgrenze lebende Bevölkerung. Ihre Sichtweise entspricht der Haltung der NGO, die der Frage nachgehen, welche Bedingungen erfüllt sein müssen, damit die PPP auch wirklich auf die Entwicklungsprobleme eingehen, die sie lösen sollen. Sie weist auf die inhärenten Widersprüche des Konzepts hin und bezweifelt die Fähigkeit, die möglichen Divergenzen zwischen den Interessen der privatwirtschaftlichen Unternehmen und den Aufgaben der öffentlichen Hand zu überwinden. So zeigt die Autorin anhand von Beispielen (Bau von Sozialwohnungen in Südafrika, Staudamm in Uganda sowie Wasserversorgung und Abwasserentsorgung in El Alto, Bolivien) die Grenzen der PPP im Infrastrukturbereich, insbesondere in Bezug auf den Zugang für die Armen, auf. Ihrer Meinung nach kann das PPP-Modell nur erfolgreich sein, wenn es als offener Prozess verstanden wird, der die Interessen aller Akteure berücksichtigt. Sie illustriert ihre Aussage am Fall einer bolivianischen Genossenschaft, welche die Wasserversorgung der Stadt Santa Cruz sichert.

Die Privatisierung der öffentlichen Wasserversorgung wird am Beispiel des Nigers erläutert: Mahaman Tidjani Alou beleuchtet die Rolle des Staates bei der Wasserbewirtschaftung sowie das Auftreten neuer, nichtstaatlicher Akteure. Für den Autor ist die Reform im Wassersektor der Ausdruck einer Sanktion staatlicher Misswirtschaft und zeigt die Notwendigkeit auf, einen neuen Rahmen für das staatliche Handeln festzulegen, der neue, nichtstaatliche Akteure wie private Unternehmen oder Vereinigungen in Erscheinung treten lässt. Eine differenzierte Analyse der Wasserversorgung in der Stadt und auf dem Land erlaubt die Darstellung einer vielförmigen Logik mit diversen Umsetzungsmodalitäten je nach den sehr unterschiedlichen sozioökonomischen Gegebenheiten zwischen Stadt und Land.

Der Artikel von Sabine Hoffmann befasst sich im Zusammenhang mit dem Begriff „Partnerschaft“ mit den institutionellen Regimen von Eigentum und

1 Es handelt sich um die Artikel von Frédéric Lapeyre, Werner Gruber und Dieter Rothenberger, Xavier Tschumi Canosa, Christine Eberlein, Mahaman Tidjani Alou, Sabine Hoffmann. 
Besitz. Nach Ansicht der Autorin werfen die Partnerschaften unweigerlich Fragen der institutionellen Rahmenbedingungen auf, welche die Rechte, Pflichten, Privilegien und „Nichtrechte“ der betroffenen Akteure festlegen. Der Artikel zeigt die verschiedenen Logiken auf, denen die Akteure im Rahmen von Partnerschaften unterworfen sind: ökonomische Rationalität (im Zusammenhang mit einer auf das Eigentum ausgerichteten Wirtschaft) oder ökosoziale Vernunft (im Zusammenhang mit einer auf den Besitz ausgerichteten Wirtschaft). Welcher Logik folgen die Akteure? Die Antwort der Autorin stützt sich auf eine Fallstudie aus Cochabamba in Bolivien über den Zugang zu Trinkwasser.

Gilles Carbonnier geht die Problematik der PPP unter dem Gesichtspunkt der Privatisierung öffentlicher Dienstleistungen in bewaffneten Konflikten an. Angesprochen werden aus juristischer und humanitärer Sicht die Entstehung privater Militärfirmen, aber auch die Privatisierung und Auslagerung der Wasserversorgung, der Führung von Haftanstalten sowie der Gesundheitspflege. Drei Hauptfragen werden gestellt: Wie vereinbar ist das Ziel der Gewinnmaximierung der privaten Unternehmen mit dem Streben des Staates nach Wahrung von öffentlicher Sicherheit und Gesundheit? Wie kann die Einhaltung des Völkerrechts durch private Unternehmen gefordert werden, und welche Verantwortung trägt der Staat, wenn diese Firmen gegen das humanitäre Völkerrecht verstossen? Gibt es echte wirtschaftliche Beweggründe für Privatisierungen als Hauptargument der politischen Verantwortlichen, die sich dafür einsetzen? Die Antworten auf diese Fragen hängen davon ab, wie die humanitären Organisationen ihre komplexen Beziehungen zu den privaten Akteuren verstehen.

Stefanie Koch präsentiert die Motivationen und Praktiken des privatwirtschaftlichen Unternehmens Holcim. Bei dieser Firma handelt es sich um einen der grössten Zement- und Betonlieferanten weltweit. Die Autorin weist darauf hin, dass die Partnerschaften mit mehreren Akteuren „multisektoriell“ sind und folglich verschiedene, möglicherweise sogar widersprüchliche, Motivationen integrieren müssen. Sie unterscheidet im Privatsektor zwischen strategischen und operationellen Partnerschaften. Im ersten Fall geht es um die Organisationen, mit denen ein Unternehmen eine langfristige Beziehung aufbauen will: Entwicklungsagenturen oder Nichtregierungsorganisationen. In einem ersten Schritt, der „Vorstudienphase“, können die möglichen Partner identifiziert und die jeweiligen Motivationen abgeklärt werden. Ausserdem ist zu entscheiden, ob die verfolgten Ziele vereinbar sind und ob die Zusammenarbeit bei der Erarbeitung des Projekts konkretisiert werden kann. Bei den operationellen Partnerschaften ist die erste Phase erfolgreich abgeschlossen worden. Der Partner ist identifiziert, die Ziele sind festgelegt, und man geht zur operationellen Phase des gemeinsamen Projekts über. Diese Ausführungen werden anhand einer Reihe von PPP zwischen der Firma Holcim und verschiedenen Partnern erläutert. Die Autorin geht auf die zahlreichen potenziellen Vorteile der PPP ein, äussert sich jedoch vorsichtig zum Risiko, dass etwas nicht planmässig verlaufen könnte, wenn der Partner nicht geeignet ist. 
Der vierte Teil des Werks, „Hintergrundinformation“, enthält eine reichhaltige Bibliographie mit rund zwanzig detailliert und kritisch präsentierten Dokumenten, Werken oder Referenzartikeln. Judith Richter legte den Akzent besonders auf Dokumentenquellen aus internationalen Organisationen, insbesondere den Vereinten Nationen, der Weltbank und dem Internationalen Währungsfonds, die das neue Paradigma der Entwicklungszusammenarbeit für eine stärkere Beteiligung des Privatsektors bestimmen. Neben diesen Berichten führt Judith Richter auch die bedeutendsten Autoren auf, die kritische Werke über die neue Ausrichtung der internationalen Organisationen veröffentlicht haben. Auf diese Weise werden die LeserInnen auf zusätzliche Dokumentenquellen aufmerksam gemacht, die sich mit einer von den Artikeln dieses Dossiers nur wenig angesprochenen Dimension der PPP befassen.

\section{Blickpunkt}

Am Ende dieser Einführung, und bevor der Leser und die Leserin die zahlreichen Facetten der PPP kennen lernt, sei darauf hingewiesen, dass es wichtig ist, die Anzahl der in der Entwicklungszusammenarbeit tätigen Akteure zu erhöhen. Entwicklung ist in der Tat eine Aufgabe des öffentlichen und des privaten Sektors. Alle müssen sich gemäss den Millenniums-Entwicklungszielen für den Kampf gegen die Armut engagieren. Die neuen Bündnisse in Form von PPP müssen jedoch eine Reihe von Voraussetzungen erfüllen, wenn sie zur Erreichung dieser Ziele beitragen wollen.

Unsere Überlegungen bei der Erarbeitung dieses Dossiers wurden unter anderem von der Frage nach den Bedingungen geleitet, unter denen die PPP eine positive Wirkung entfalten und gleichzeitig unerwünschte Folgen begrenzen können. Es ist zwar schwierig, einen genauen Überblick über die möglichen Massnahmen zu geben, jedoch zeigen die von den verschiedenen Autoren in ihren Beiträgen gegebenen Antworten einige Lösungsansätze auf, die nachfolgend kurz zusammengefasst sind: 1. die Bedingungen für die Ausrichtung der öffentlichen und privaten Interessen zugunsten der Entwicklung; 2. die von den Partnern festgelegten Leitlinien zur Klärung ihrer Zielsetzungen; 3. die Notwendigkeit eines verbindlichen juristischen Rahmens für die verschiedenen Akteure; 4. ein besonderer Akzent bei den Empfängern der Dienstleistungen, die für eine PPP in Frage kommen; und 5. die bei der Verwirklichung einer PPP unerlässlichen Regulierungsorgane.

Die grundlegende Frage, inwiefern die PPP wirklich ein nachhaltiges Modell zur Verknüpfung der privaten und öffentlichen Interessen für die Entwicklung darstellen können, führt zu den Beweggründen der Akteure. Wie der Rahmentext 4 zeigt, sind die Motivationen der Partner oft unterschiedlich. Damit stellt sich die Frage nach dem Kräfteverhältnis zwischen privatem und öffentlichem Sektor. Wenn der Privatsektor durch multinationale Unternehmen mit sehr viel mehr Macht als die Partnerländer vertreten wird, gibt die Wahrung der öffentlichen Interessen zu grosser Sorge Anlass. Es ist jedoch auch unbestritten, dass sich auf lokaler Ebene Partnerschaften zwischen Kleinunternehmen und der öffentlichen Hand als Gewinn bringend erweisen können, sofern die Behörden 
in der Lage sind, einen klaren vertraglichen Rahmen zugunsten von Zielen im allgemeinen Interesse durchzusetzen.

\section{Rahmentext 4}

\section{Übersicht über die Beweggründe der Partner}

Welches Interesse hat der Privatsektor an einer PPP?

1. Verkauf der Güter und Dienstleistungen, die das oberste Ziel der fraglichen privaten Einrichtung darstellen:

1.1. zur Erzielung eines Einkommens

1.2. zur Gewinnmaximierung

2. Erweiterung des Marktes

3. Abschottung des Marktes

4. Einflussnahme auf den politischen und juristischen Rahmen, in dem das Unternehmen tätig ist

5. Verbesserung des Markenimages.

Welches Interesse hat der öffentliche Sektor an einer PPP?

1. Kostengünstigere Versorgung, weil

1.1. der Privatsektor effizienter sein kann, da der Markt zu wenig Gelegenheiten bietet oder zu unbedeutend ist, dass die öffentliche Hand investiert;

1.2. die privaten Unternehmen weniger Sachzwängen bei Entscheidungen unterliegen, die hohe Haushaltsausgaben verursachen (insbesondere in Bezug auf die Beschäftigungsbedingungen).

2. Ausweg aus anderen politischen oder juristischen Sachzwängen

3. Förderung der Innovation

4. Bessere Nutzung der technischen Fachkenntnisse privater Unternehmen

5. Entfernung gewisser Posten aus der Staatsrechnung (z.B. Übertragung von Schulden der öffentlichen Verwaltung auf die neuen PPP).

Die Autoren des Dossiers stellen fest, dass die Beteiligung des Privatsektors an der Verfolgung der Entwicklungsziele keine Selbstverständlichkeit ist und zahlreiche Fragen aufwirft. Jeder legt seine Ideen zum Abbau der Spannungen oder zur Behandlung von Interessenkonflikten dar. Das Werk präsentiert die verschiedenen Sicherheitsmassnahmen oder Leitlinien der privaten und öffentlichen Akteure. Alle Entwicklungsagenturen der Schweiz, Frankreichs und Deutschlands sowie auf lokaler Ebene des Kantons Genf und das Privatunternehmen Holcim, deren jeweilige Erfahrungen in diesem Dossier angesprochen werden, haben einen Bezugsrahmen für ihr Vorgehen im PPP-Bereich geschaffen. Dieser Schritt scheint ihnen nötig oder gar unverzichtbar, um ihre Ziele und Beweggründe zu klären und die erwarteten Ergebnisse festzulegen. Solche Dokumente dienen oft als Grundlage für die Gespräche zwischen den verschiedenen Partnern, die an einem gemeinsamen Projekt arbeiten. In der kommentierten Bibliographie legt Judith Richter den Schwerpunkt besonders auf die Massnahmen der Vereinten Nationen und bestimmter Sonderorganisationen zur Verabschiedung von Richtlinien über die Zusammenarbeit zwischen den internationalen Organisationen und den privaten Unternehmen, um die öffentlichen Interessen im Rahmen von PPP zu wahren.

Einseitige und freiwillige Leitlinien scheinen unverzichtbar, um die Ziele der betroffenen Akteure zu klären, und einige halten auch einen verbindlichen juristi- 
schen Rahmen zur Regelung der Tätigkeit von Unternehmen für notwendig. So begrüssen Peter Ulrich und Florian Wettstein die Idee einer Formalisierung der Beziehungen zwischen dem Privatsektor und den Vereinten Nationen. Dies soll insbesondere über den Global Compact als ,unbefristete Plattform gemeinsamen Lernens zur Weitereintwicklung der Standards guter Unternehmensführung“ geschehen. Die Autoren stellen jedoch auch die Frage nach der Glaubwürdigkeit einer solchen Übung und schlagen die Einführung von Mechanismen zur Überprüfung und Durchsetzung der zehn Prinzipien des Global Compact vor. Sie gehen sogar noch einen Schritt weiter: Wenn die am Global Compact beteiligten Unternehmen wirklich ihren „Bürgersinn“ beweisen wollten, müssten sie mehrheitlich dem zurzeit von der Menschenrechtskommission diskutierten Entwurf zu Normen über die Verantwortung der transnationalen Gesellschaften und anderen Unternehmen im Bereich der Menschenrechte zustimmen.

Viele Autoren erinnern daran, dass der PPP-Begriff nicht nur die Beziehungen zu den Unternehmen umfasst, sondern dass die neuen Partnerschaften auch die Beteiligung der Bevölkerung an diesem Prozess berücksichtigen müssen. Frédéric Lapeyre steckt den allgemeinen Rahmen für die Beteiligung der Bevölkerung ab. Diese Notwendigkeit wird im Übrigen von zahlreichen Autoren (Christine Eberlein, Mahaman Tidjani Alou, Sabine Hoffman und Gilles Carbonnier) anhand konkreter Beispiele bekräftigt. So wird die Frage nach der Rolle der Zivilgesellschaft und der Nutzer der Dienste gestellt, die für eine PPP geeignet sind. Bei einer Entwicklung, die darauf abzielt, die schwächsten Teile einer Bevölkerung aus der Armut zu führen, müssen die Betroffenen auch zu Wort kommen, weil sie die angemessensten Lösungen besitzen. Eine solche Beteiligung erfordert jedoch ebenfalls genaue Regeln. Dieser Lösungsansatz bedeutet, dass die für die Entwicklung nützlichen PPP nicht als Partnerschaft zwischen zwei Akteuren (Staat und Unternehmen), sondern zwischen drei Akteuren, das heisst unter zusätzlicher Beteiligung der Benutzer, zu sehen sind.

Wir müssen feststellen, dass neue Bündnisse wie die PPP die Praktiken der Entwicklungszusammenarbeit unbestreitbar komplizierter gestalten. Die Beiträge des Dossiers haben gezeigt, dass die Umsetzung von Projekten durch Partner mit unterschiedlichen Beweggründen oder Interessen einen Dialog und eine Regulierung erfordert. Regulierungsorgane, deren Form und Praxis von einer PPP zur anderen variieren, scheinen unerlässlich, insbesondere um die oft divergierenden und von sehr heterogenen Akteuren vertretenen Interessen unter einen Hut zu bringen. Diese Regulierungsarbeit muss zu einer Verbesserung der Entwicklungsperspektiven im allgemeinen Interesse führen. Der Artikel von Mahaman Tidjani Alou bietet diesbezüglich einen viel sagenden Einblick in die Schwierigkeiten, die bei einer solchen Übung auftreten können.

Mit der Veröffentlichung dieses Werks möchten wir ein neues Konzept klären und die Chancen, aber auch die Grenzen eines Entwicklungsinstruments aufzeigen, das von den Entwicklungsagenturen immer stärker genutzt wird. Die verschiedenen Beiträge im Dossier enthalten Lösungsansätze, die alle weiter vertieft werden müssten. Wir hoffen jedoch, dass die so angestossene Diskussion fortgesetzt wird und zu einer Stärkung des institutionellen Rahmens für öffentlich-private Partnerschaften führt, damit diese positive Wirkungen zugunsten der ärmsten Menschen unserer Welt entfalten können. 